\title{
enREDando y conectando: Comunicacion y redes sociales
}

Ilia E. López Jiménez*

Recibido: 19 de octubre de 2009

Aceptado: 15 de noviembre de 2009

\section{RESUMEN}

En esta publicación se propone el análisis de las redes sociales desde una perspectiva comunicacional: por su contenido o temática; por su composición (cerrada o abierta), por la interacción (interna o externa) existente; por su difusión y alcance (local o internacional). Se han seleccionado tres sitios de la web que presentan características diversas: una red de autogestión comunitaria, una red académica y una red sociopolítica.

Palabras clave: Planificación estratégica de las instituciones públicas en la cuestión de género

* Universidad de Puerto Rico, Recinto de Río Piedras. E-mail: ielopez@uprrp.edu 


\title{
Tangling and connecting: communication and social networks
}

\begin{abstract}
This article is intended to make an analysis of social networks from a communicational point of view: based on their contents and topics; based on their components (closed or open); based on their existing interaction (internal or external); based on their kind of release and scope (local or international). Three websites showing several characteristics have been selected: a community self-management network, an academic network, and a sociopolitical network.
\end{abstract}

Key words: Communication, social networks, websites, academic networks. 
Social Colligato, Blog de José González Corrales ${ }^{1}$, dedicado al análisis de las redes sociales, expone en marzo de 2008 que no son las coordenadas geográficas las que marcan nuestra posición dentro de las comunidades sino las coordenadas relacionales. El análisis de las redes y sus relaciones se ha convertido en objeto de estudio de múltiples disciplinas y abarca distintos campos del conocimiento académico, tales como la estadística, la matemática, la antropología, la sociología, la psicología, las ciencias políticas. Del mismo modo que existen las redes de computadoras, las redes sociales conectan a individuos y organizaciones vinculados por lazos de amistad, académico, financiero, laboral, político; todos vinculados mediante el intercambio de información.

Microfoft explica en la publicación electrónica Qué son y cómo funcionan las redes sociales: "El concepto de red social, creado por la antropología inglesa para superar análisis estructurales obsoletos, parte de un abstracto, esto es, tomamos un punto de partida de estudio y establecemos las distintas relaciones entre los individuos. Para cada punto de partida crearemos distintas redes y, por supuesto, modos de relación. Este concepto, tan básico como sencillo es lo que hace de las redes sociales un mundo espectacularmente útil y ampliamente difícil de abarcar" ${ }^{2}$.

Así, por ejemplo, Duncan Watts, director del Small World Project ${ }^{3}$, se propone estudiar las relaciones entre los usuarios del correo electrónico. De igual modo, Karina Helbert ${ }^{4}$ (2003) utiliza la teoría de redes de contacto basada en la creación de nodos de información. La creación de dichos nodos se fundamenta en la teoría de algoritmos y se ha aplicado a distintos estudios de comunicación escrita, en particular en el estudio de las cartas en cadena chain letters. Halbert comenta

1 http://josegonzalezcorrales.wordpress.com

2 http://www.microsoft.com/spain/empresas/rrpp/redes_ sociales intro.mspx

3 http://smallworld.columbia.edu/description.html

4 http://www.psp-sa.com/News_SugNo2.htm sobre el desarrollo teórico de la teoría de redes y cómo ha permitido constatar que estos contactos siguen una tipología de nodos en los que influyen la calidad y el tipo de conexiones existentes. Es decir, las oportunidades de lograr nuestro objetivo comunicacional en la red dependerán en gran medida de cuántos contactos se tengan y cuán bien conectados estén los mismos. Al fortalecer cualquier parte de la topología de la red, se está reconfigurando el conjunto de relaciones y el efecto que se logre será mayor.

Adrían Coutín Domínguez, ${ }^{5}$ mediante su página Web, sobre los trabajos que se pueden realizar en el análisis de redes sociales, menciona los siguientes: los patrones existentes en los vínculos entre personas, organizaciones, naciones, y cualquier tipo de comunidad que se relacione por un asunto de interés común, la circulación de los nodos de información a través de vínculos directos o indirectos en las redes, cómo se adquieren los recursos disponibles en las redes y cómo se transfieren dichos recursos. Coutín (2003) define como unidad de análisis la relación que se establece entre los integrantes de una comunidad.

Steve Borgatti (2003), ${ }^{6}$ además de la relación social, centra su atención en la estructura del contenido, pues cada relación genera una estructura diferente y tiene diferentes implicaciones. Según Borgatti, los siguientes aspectos definen el análisis de redes sociales (ARS): el fenómeno estudiado, la perspectiva adoptada, las metodologías y las herramientas utilizadas. Partiendo de dicha clasificación, se podría integrar el análisis de las herramientas, los recursos intercambiados utilizando la computadora como medio de comunicación, tales como los documentos electrónicos, los programas. Desde ese punto de vista, se concibe el sitio web como una relación, y dentro de ella, se pueden estudiar los contenidos que son intercambiados por la comunidad y los servicios

5 http://www.acoutin.com/publications.htm

6 www.analytictech.com/networks/introduccion2.pdf 
que se pueden brindar a una comunidad o a otras comunidades.

Si bien la conexión interpersonal surge de manera espontánea, en ocasiones se presenta de manera estructurada como una estrategia comunicativa destinada a reforzar la presencia en la red para cumplir un objetivo comunicativo determinado. Desde ese punto de vista, la red social se transforma en una estrategia de mercadeo. Tal es el caso del uso de You Tube como promoción dentro del mercado laboral para presentar el Vídeo Currículum ${ }^{7}$. El Blog Salmón ${ }^{8}$, dedicado a la economía y las finanzas, publicó recientemente Usa las redes de contacto para acceder a tu nuevo empleo. En dicha publicación se recomiendan redes profesionales tales como LinkedIn, Viadeo, pero también otras como Facebook. Viadeo, por ejemplo, se promociona como una "red que posibilita tener una identidad digital multilingüe y ganar notoriedad'9. El Centro Virtual de Búsqueda de Empleo, Puerto Rico, ofrece el siguiente consejo: "Comparte esta página con tus amigos. Nunca sabes si alguien está pasando por la experiencia de estar desempleado y no quiere decirlo, [tal vez] le llegue a alguien que conozca de alguien que lo esté, o alguien que no esté satisfecho con su empleo actual y quiera cambiarlo". Livia García Faroldi en Redes sociales y mercado de trabajo (2007, diciembre, p. 7), indica al respecto que dada la menor utilidad que tiene hoy un título escolar, debido a la multiplicación de títulos existente, la inversión en relaciones se vuelve más rentable. Según García Faroldi, "El capital relacional depende de las posiciones relativas en las que un individuo pueda encontrarse, pues el ocupar una posición estratégica en una red de relaciones manifiesta la posibilidad de tener más o menos información sobre situaciones determinadas".

7 http://www.carlosleopoldo.com/post/como-hacer-uncurriculum-que-impresione-curriculum-en-video

8 http://www.blogsalmon.com

9 http://www.viadeo.com/es/connexion
En la publicación electrónica de Microsoft, Qué son y como funcionan las redes sociales, mencionada previamente, se expone: "En sociedades complejas el individuo debe manejar tres tipos de intercambio (reciprocidad, redistribución y mercadeo); ello implica que participa simultáneamente de los tres tipos de relaciones sociales: una relación de confianza, una de jerarquía y otra de clase" ${ }^{\prime 10}$. De esta manera se percibe la red social como una representación de las relaciones entre las personas o entre las organizaciones en donde los individuos con un grado alto de cercanía tienen una relación más estrecha con otros individuos y sostienen vínculos fuertes que incluyen intimidad, provisión mutua de servicios y contacto frecuente. Aquél cuya relación es intermediaria funciona como un punto de conexión que puede intervenir favorablemente en las relaciones, pero al mismo tiempo puede generar huecos que desconectan individuos o redes completas. Los vínculos débiles representan relaciones distantes con escaso contacto y limitada retroalimentación.

La multiplicidad representa la cantidad de relaciones que convergen en un vínculo. Mientras más relaciones existan, mayor es la multiplicidad. Se debe tomar en cuenta que la existencia de vínculos débiles es necesaria para difundir información, puesto que los intermediarios funcionan como conectores de segmentos que de otra manera estarían aislados. De esta manera, los vacíos o agujeros estructurales son llenados por individuos cuyas relaciones cruzan estos agujeros (García Faroldi, 2007). Indudablemente, el conocer dónde están los vacíos y establecer conexiones entre ellos representa una ventaja competitiva tanto para los individuos como para las organizaciones.

Es pertinente considerar la propuesta de José Manuel Gaete Fiscelia y Jorge Ignacio Vásquez formulada en REDES, Revista Hispana para el Análisis de Redes Sociales. Dicha propuesta define tres

10 http://www.microsoft.com/spain/empresas/rrpp/redes_ sociales_intro.mspx 
dimensiones del capital social como determinantes en la creación y producción de conocimiento mediante redes sociales, dimensiones pertinentes al momento de identificar la importancia de los agujeros estructurales: (Gaete Fiscelia, Vásquez, 2008, junio, p. 9).

1. La conectividad indica los recursos que se encuentran al alcance del individuo, la posición que el individuo tenga en la red será primordial a la hora de acceder los recursos que circulan. La conectividad aporta grandes beneficios informativos para aquellos nodos que sean puente o conecten sectores de la red que carecen de relaciones.

2. La coordinación facilita la integración, acceso y selección de los recursos que se encuentran en la red. Esta integración se determina por las conexiones e los individuos en la red.

3. La motivación proporciona la compensación para los individuos que se implican en el proceso y en la red. De esta manera, el hecho de formar parte de cierta red puede motivar a los individuos que la componen.

Partiendo desde lo anteriormente expuesto, se propone el análisis de las redes sociales desde una perspectiva comunicacional: por su contenido o temática; por su composición cerrada o abierta, dependiendo de la interacción y de la comunicación interna o externa existente; por su difusión y alcance local o internacional. La hipótesis de trabajo parte de la siguiente propuesta formulada en la revista electrónica Razón y Palabra", "la comunicación ha abandonado de manera general un punto de vista necesario acerca de las estructuras de las relaciones sociales que conforman los grupos sociales y extensas redes de intercambio, interacción o interrelación". De esta manera se concibe "un problema estructural de vínculos y generación de ciertos conglomerados sociales de sentido a partir de los intercambios

11 http://www.razonypalabra.org.mx/n6l/gvelez.html realizados entre sujetos y organizaciones que puedan ser expresiones de estructuras de relaciones efectivas más generales de intercambio" (Vélez G. 2008, agosto, p. 1). En consonancia con dicha propuesta, Manuel Castells aborda la dimensión cultural; es decir, el sistema de valores, creencias y formas de construir mentalmente una sociedad es decisivo en la producción y las formas de Internet, y propone las cuatro capas culturales de Internet: la universitaria, la de los hackers, la de las formas culturales alternativas y la empresarial.

En primer lugar, se abordará el análisis una red dedicada a la autogestión comunitaria, Casa Pueblo de Adjuntas, Puerto Rico. Casa Pueblo se define como "Una organización de autogestión comunitaria que promueve a través de la iniciativa individual y colectiva la participación voluntaria que propone y desarrolla alternativas para proteger el ambiente, afirmar los valores culturales y humanos". La conexión de la red social de Casa Pueblo se establece mediante la publicación de una lista de correo electrónico y la página Web www.casapueblo.org; actualmente su difusión tiene alcance internacional. La creación de dicha publicación y su difusión a través de la Web llenó un vacío estructural existente en relación con la autogestión comunitaria y la comunicación para el cambio social. La lista de correo posibilitó el uso del correo electrónico como canal de comunicación rápido para el intercambio de información. El objetivo general de Casa Pueblo, expresado en la Página Hogar de esta organización comunitaria, es el siguiente: "Unir a distintos sectores de la comunidad para luchar sobre aquellas cosas que les afectan, proponer alternativas social y ecológicamente justas e implementarlas". Dicha red se concibe como una red abierta que utiliza estrategias del mercadeo social12 para promulgar

12 Social marketing was "born" as a discipline in the 1970s, when Philip Kotler and Gerald Zaltman realized that the same marketing principles that were being used to sell products to consumers could be used to "sell" ideas, attitudes and behaviors.

http://www.social-marketing.com/Whatis.html 
el desarrollo sustentable, y ha establecido en la actualidad mayor apertura hacia otros sectores y conexiones con otras redes de autogestión comunitaria mediante la presencia en Facebook, estrategia de comunicación corporativa utilizada por otras empresas y organizaciones que buscan crear y aumentar las conexiones entre los usuarios. De esa manera se incrementa la multiplicidad mediante la interconexión de vínculos tales como Radio casa Pueblo. Si se toma en cuenta el contexto de la red, el comportamiento de los actores incide en las redes personales, y estos actores pueden, a su vez, seleccionar sus relaciones e influir sobre ellas. Es esta la razón por la cual encontramos doble presencia en la red, destinada a aumentar la conectividad. En relación con el networking, la red de contactos entre Pequeñas y Medianas Empresas (pymes) Microsoft13 aconseja lo siguiente:

De esta forma se genera una "tela de araña" entre pymes en la que, para lograr que se hagan las cosas, usted no cuenta con el poder jerárquico. Usted solo cuenta con el poder personal, con su talento para gestionar las relaciones profesionales. Un aspecto a favor de la red de Casa Pueblo es la motivación de los actores que la componen y la coordinación demostrada a través de la información constante que fluye; dichos factores han colaborado en el fortalecimiento de la imagen. Así, Microsoft (p.2) comenta sobre la imagen en la network "Generar una imagen de éxito positiva y real es muy importante.

En segundo lugar, se propone el análisis de las redes académicas a partir del análisis realizado por Gaete Fiscella e Ignacio Vásquez (2008, junio), en relación con la estructura académica vista desde el análisis de las redes sociales. En dicho estudio, Conocimiento y estructura en la investigación académica: una aproximación desde el análisis de las redes sociales, se elabora un "mapeo" de redes relacionadas con

13 Networking: las cosas ocurren cuando alguien quiere que ocurran. Disponible en: http://www.microsoft.com/spain/ empresas/rrpp/networking.mspx la investigación académica. Se destaca también la importancia de proveer mecanismos de carácter informal o social como medios de transmisión del conocimiento tácito y particular (p. 2). El conocimiento tácito o implícito es aquel que poseen las personas y que no puede extraerse fácilmente. El Colegio invisible hace referencia a un círculo social cuyos integrantes generalmente conocen a una parte del mismo, pero son influidos por otros integrantes con quienes no están conectados directamente. Es en este contexto informal donde surgen vías de interacción anónima e implícita que posibilitan la creación del conocimiento y su circulación (p. 7). Es indispensable para la difusión de ese conocimiento la creación de interacciones personales de modo tal que se haga visible lo invisible. Así se constata que la creación de conocimiento no parte únicamente del establecimiento de vínculos estrechos, sino de la capacidad para establecer relaciones fuera del grupo de pertenencia. La creación de una red amplia en tamaño, pero cerrada en su composición, tiende a ser egocéntrica y dificulta el flujo de la comunicación externa. Al contrario, la red abierta tiende a ser socio-céntrica y facilita el flujo de la comunicación externa. A mayor cohesión entre los miembros del grupo, mayor será la posibilidad de difusión de la comunicación interna y de la externa. Si observamos, desde el punto de vista institucional, la tipología de las redes existentes en el Recinto de Río Piedras de la Universidad de Puerto Rico, se constata que existen diversos recursos empleados como modos de comunicación. La comunicación interna en los departamentos y en las facultades, por su naturaleza, tiende a ser cerrada y egocéntrica, se transmite mayormente a través del correo electrónico, medio utilizado para la comunicación formal y la informal, en particular para la difusión del rumor, los llamados "rumores de pasillo". No existen redes de conexión que permitan enlazar investigadores de las diferentes facultades o redes de conexión entre los investigadores internos y externos del Recinto. La presencia en la red se ha dado mayormente a través de la página web, tanto 
en los departamentos, las facultades y escuelas como en las dependencias administrativas del Recinto. Actualmente, algunas facultades tales como Administración de Empresas, la Escuela de Comunicación Pública cuentan con una presencia en Facebook; sin embargo, todavía el alcance es limitado. ${ }^{14}$ Desde esa perspectiva, se constata que en el ámbito académico, las redes constituidas no varían mucho en función de su composición y tal como concluyen Gaete Fiscella y Vásquez (2008, junio, p.29): "De los agujeros estructurales que existen, los grupos intermediados son principalmente de investigadores externos, lo que entrega a los intermediadores un gran poder y control del flujo de información no redundante o distinta de la que se genera en los proyectos de los departamentos". La posición académica no determina ni, menos, asegura, un posicionamiento relevante y central en una red donde circulan intangibles como la información y el conocimiento". El posicionamiento en la red se determinará en gran medida por la capacidad institucional de crear y establecer lazos entre la comunidad externa e interna (networking interno y networking externo) ${ }^{15}$, de modo tal que se pueda gestionar y difundir el conocimiento. Para ello, es indispensable la creación de redes abiertas con vínculos fuertes, de modo tal que las redes sociales de conocimiento puedan expandirse y flexibilizarse en el mundo virtual de la sociedad del conocimiento y de la globalización. Desde ese aspecto, al conectar más de dos personas, la red provee un espacio tridimensional ideal para la creación del capital intelectual.

Participar en una red social, trabajar en Network implica aplicar principios distintos de cuando se trabaja internamente. Dichos principios suponen lo siguiente: las relaciones se establecen

14 La matrícula de la FAE es 2,300 y cuenta con 420 amigos en Facebook.

15 Microsoft ilustra tres situaciones: Networking interno, Networking entre empresas (organizaciones) de una misma categoría y Networking desde una empresa (organización) grande hacia fuera. http://www.microsoft.com/spain/ empresas/rrpp/networking.mspx entre iguales sin que medie el poder jerárquico. La capacidad y el talento para manejar la comunicación, la empatía y las relaciones profesionales constituyen el factor clave que determinará el éxito de la red. El área de influencia va mucho más allá de los límites geográficos, pues el espacio en la red no tiene fronteras. De igual manera, se pueden recibir contactos desde mucho más lejos. La proactividad constituye un factor decisivo, ya que hay que descubrir nuevas maneras de hacer las cosas, ser capaz de liderar el cambio y reducir las resistencias ${ }^{16}$. Tejer y mantener una red de contactos exige tiempo y esfuerzo. El funcionamiento de la misma comienza cuando un grupo de iniciadores invita a un grupo de amigos y conocidos a unirse a la red social, cada nuevo miembro trae consigo un grupo de miembros y el crecimiento del grupo se va agrandando por medio de enlaces entre sí. Un ejemplo típico de ese tipo de funcionamiento es la constitución del grupo temático llamado Los e-mails de Jacinta, originado inicialmente mediante el correo electrónico y posteriormente, convertido en página en la red de Facebook bajo el seudónimo de Jacinta Marín y el grupo de amigos mencionado. La red social de temática política conjugada permite a este ente anónimo romper el aislamiento sociopolítico a través de la interacción y la popularidad de un personaje ficticio. A continuación reproduzco parte de la historia de Jacinta Marín:

Mi historia es como la de tantas otras mujeres de Menudo para Atrás que fuimos casadas con hombres que nunca nos dejaron opinar de política. En el caso que me ocupa, estuve matrimoniada con el ahora difunto Agapito que Dios lo tenga en la gloria entretenido, porque si ve que me he convertido en una politóloga sata es capaz de presentarse y jalarme los pies. Cuando el murió yo quedé como tantas otras viudas de mi generación, desconsolada pero un chin aliviada. El Señor me reprenda. Porque resulta y acontece que vi como un guindou of oportiuniti de apren-

16 http://www.microsoft.com/spain/empresas/rrpp/ networking.mspx 
der lo que no me habían dejado, que era la historia de mi país y los acontecimientos de la política.

La intervención de Jacinta en la red constituye un intento reflexivo, generador y organizador de interacciones, cuyo sujeto se funda a sí mismo y se diferencia de los demás por su lenguaje cuasi fonético, por el uso de expresiones coloquiales propias de la ruralía, del habla del ya casi desaparecido campesinado puertorriqueño, por la inclusión del spanglish y por su aproximación a la sátira política que abarca sin distinción a todos los partidos. Intervención que muestra el siguiente ejemplo:

La Virgen proteja a la pobre abuelita roja. Me dicen que en los joums ya están formándose las peleas entre azules y coloráos. Así es que no te extrañe que pronto veremos anoder viejito, meciéndose también en un sillón, y contestándole a abuelired en inglés. Pero pasa que todavía don Fortu no está seguro si at dis moument, con la fresquedad del recuerdo del teikover que le hizo don Primitivo en la cámara, le conviene decir que rajen la papeleta para que metan a todos los suyos. Te mantendré pousted para que no te pierdas en medio de este reperpero. iLa vida es compleja en Borinquen!

Así, por medio de lo "real maravilloso" Jacinta va transformando el tejido social de la red, y dicho tejido se va reconstruyendo con la aportación de cada suscrito a la red, cada nuevo miembro que ingresa va transformando la constitución del tejido. Dicha red social de tendencia circular ${ }^{17}$ se ha transformado en una red abierta que cuenta en la actualidad con miembros dentro y fuera de la Isla. Así, la propuesta de construcción de redes sociales se enmarca por la construcción de un sujeto definido por sus intercambios entre actores sociales, construyendo convivencia y solidaridades mediante relaciones directas e indirectas ${ }^{18}$.

17 El centro de la red es ocupado por una persona.

18 Existe una relación directa cuando una persona brinda apoyo a otra en el marco de la relación existente en la Web.
Como dice Jacinta "guey to go".

Los tres tipos de análisis de redes presentados constituyen una aproximación al análisis de redes sociales desde un punto de vista comunicativo. A modo de conclusión, se propone el análisis de las redes vinculado a su contenido, a su temática y su modo de relación; se presentan las siguientes recomendaciones:

- Desarrollar vínculos organizacionales que se expresen en vínculos electrónicos.

- Promover vínculos temáticos e incluir la participación de expertos encargados en fortalecer los vínculos.

- Crear multiplicidad de relaciones a través de la Web 2.0 (Twitter, Facebook), listas de correo.

Sin embargo, se debe destacar, además, el espacio Web dedicado al análisis, tal como el editorial de Maestros del Web ${ }^{19}$ dedicado a las redes sociales y que presenta una recopilación de redes sociales de habla hispana. Asimismo, Prácticas grupales. Un modelo de trabajo en redes, contiene una sección destinada a las redes sociales ${ }^{20}$.

De esa manera, se va tejiendo la red social, se van enredando y conectando la relaciones que van a llevarnos por los caminos del mundo real y del mundo virtual.

\section{REFERENCIAS BIBLIOGRÁFICAS}

Aúnsolo, A. (2008) Qué son y cómo funcionan las redes sociales: Introducción. Microsoft. Centro para Empresas y Profesionales. Fecha de consulta: 12 de mayo de 2008. Disponible en: http://www.microsoft. com/spain/empresas/rrpp/redes_sociales_intro. mspx

\footnotetext{
19 http://www.maestrosdelweb.com/editorial/redessociales

20 http://www.practicasgrupales.com.arhttp://www. maestrosdelweb.com/editorial/redessociales
} 
Borgatti, S. (2003). Conceptos básicos de redes sociales. Fecha de consulta: agosto 2008. Disponible en: http://www.analytictech.com/networks/introduccion2.pdf

Brito, E. (2008, agosto) Usa las redes de contacto para acceder a tu nuevo empleo. El blog salmón.com. Fecha de consulta: agosto, 2008. Disponible en: http://www.elblogsalmon.com/2008/08/06

Costa, J. Comunicación en el siglo XXI. RRPPnet. Fecha de consulta: 28 de febrero de 2008. Disponible en: http://www.rrppnet.com.ar/comsigloXXI.htm

Castells Manuel. (2002). La dimensión cultural de Internet. Fecha de consulta: agosto de 2008. Disponible en: http://www.uoc.edu/culturaxxi/esp/articles/ castells0502/castells0502.html

Coutín Domínguez, A. (2003). Arquitectura de información para sitios web. Congreso Cultura y Desarrollo. Habana, Cuba. Fecha de consulta: mayo 2008. Disponible en: http://www.acoutin.com/esp/ publicaciones.htm

Gaete Fiscella, J.M. y Vásquez, J.I. (2008, junio). Conocimiento y estructura de la investigación académica: una aproximación desde el análisis de redes sociales. REDES revista hispana para el análisis de redes sociales Vol. 14\#5. Fecha de consulta: agosto 2008. Disponible en: http://revista-redes.rediris.es

García Faroldi, L. (2007, diciembre) Redes sociales y mercado de trabajo. REDES- Revista hispana para el análisis de redes sociales Vol.13,\#10. Fecha de consulta: agosto 2008. Disponible en: http://revista-redes.rediris.es

Grossoni Mónica. Networking: Las cosas ocurren cuando alguien quiere que ocurran. Microsoft. Centro para Empresas y Profesionales. Fecha de consulta: agosto 2008. Disponible en: http://www.microsoft. com/spain/empresas/rrpp/networking.mspx

Vélez, G. (2008, agosto) Exploración de la relación entre redes sociales y comunicación. Razón y Palabra Número 61. Fecha de consulta: agosto 2008. Disponible en: http://www.razonypalabra.org.mx/ n61/gvelez.html

Zamora, M. Redes Sociales en Internet. Maestros del Web. Fecha de consulta: agosto 2008. Disponible en: http://www.maestrosdelweb.com/editorial/ redessociales 